Meta

Journal des traducteurs

Translators' Journal

\title{
Le verbe qui vole : mot de l'interprète
}

\section{Thérèse Romer}

Volume 20, numéro 1, mars 1975

Vingt ans de traduction. Bilan et prospectives

URI : https://id.erudit.org/iderudit/003975ar

DOI : https://doi.org/10.7202/003975ar

Aller au sommaire du numéro

Éditeur(s)

Les Presses de l'Université de Montréal

ISSN

0026-0452 (imprimé)

1492-1421 (numérique)

Découvrir la revue

Citer cet article

Romer, T. (1975). Le verbe qui vole : mot de l'interprète. Meta, 20(1), 36-41.

https://doi.org/10.7202/003975ar d'utilisation que vous pouvez consulter en ligne.

https://apropos.erudit.org/fr/usagers/politique-dutilisation/ 


\section{Le verbe qui vole: Mot de l'interprète}

Un anniversaire c'est, bien sûr, une célébration. On y convie parents, frères, sœurs, cousins, toute la famille. Les interprètes sont heureux de venir à la fête que représente ce numéro anniversaire, pour y retrouver les traducteurs, linguistes et professeurs qui font et qui lisent Meta. À la polonaise, nous levons le verre et disons «sto lat!», nous allumons les chandelles et chantons à la ronde «sto lat!» - cent ans! Soyez avec et parmi nous tous, Meta, heureux ensemble du chemin accompli et de celui à venir pendant cent ans encore!

Mais les anniversaires sont aussi le temps des bilans. Prenons un moment de répit, entre une conférence et une autre, entre la course d'un avion à l'autre et le centre des congrès, entre la pile des documents à lire en diagonale pour capter un sujet nouveau, et la bousculade des «verba volant 》 devant les micros ouverts. Les interprètes de conférences au Canada : d'où sortons-nous, qui sommes-nous, où nous dirigeons-nous, au sein de cette plus grande famille qui, comme nous, gagne sa vie à manier les langues?

Eh bien, tout d'abord, ce n'est pas par le nombre qu'il faut nous juger. Même là où nous sommes les plus nombreux, à Montréal et à Ottawa, nous sommes dépassés de loin par le nombre des traducteurs. Il suffit de regarder autour de soi pour s'en rendre compte. À la S.T.Q. : 850 traducteurs - et combien d'autres non organisés? Alors qu'il y $a$, en tout et pour tout, une soixantaine d'interprètes au Québec, une cinquantaine en Ontario... Dans le monde entier, quelque 1500 interprètes, contre maintes fois plus de traducteurs... C'est donc dire que nous ne pouvons pas être très largement connus, sauf dans un monde très particulier : celui des conférences, bilingues mais surtout multilingues, où nous évoluons.

Quant à la nature de notre travail, « verba volant scripta manent », c'est peutêtre par cela qu'il se distingue le plus de celui de nos confrères traducteurs. Entre des interlocuteurs qui n'emploient pas la même langue pour se parler, nous agissons comme intermédiaires, sur-le-champ, au vif des échanges. Les circonstances conditionnent les solutions. Par exemple, nous savons tous qu'un enfant peut aider le touriste étranger à demander un renseignement à l'agent de la circulation à un coin de rue ; la secrétaire peut faciliter les contacts entre son patron et l'homme d'affaires en visite ; et l'interprète d'escorte, souvent étudiant ou étudiante, fera le pont pendant une tournée industrielle ou agricole, culturelle ou touristique, qui permettra 
à un groupe de visiteurs étrangers d'apprendre à connaître un pays ou une région. Mais la communication orale la plus structurée, et souvent la plus complexe, se fait généralement lors de réunions, congrès, colloques, cours ou conférences - et c'est là que l'on fait appel à des professionnels de la «traduction » orale, les interprètes de conférence.

En ce sens, nous sommes autant sinon plus spécialistes de la communication que de la traduction. Il s'agit de faire passer-fidèlement-l'essence du message: l'idée ou l'émotion, le goût ou l'ambiance, aussi bien que le sens, au sens propre. Qui s'attache au littéral, aux équivalences lexicales, stylistiques ou linguistiques, peut laisser échapper des éléments importants du message, s'il ne sait, en plus et d'emblée, quels sont les liens et les rapports entre les êtres vivants qui se parlent, quels sont leurs cadres de référence. Dans son bagage professionnel, l'interprète doit donc avoir certaines connaissances communes avec le traducteur: connaissance approfondie des langues et des cultures qui les accompagnent, chacune ayant sa façon particulière de découper la réalité vécue. Mais d'autres aptitudes seront non moins nécessaires à l'interprète : l'oüe de la standardiste téléphonique ; la capacité de l'acteur à se mettre dans la peau d'autrui ; la voix bien placée, l'aisance de l'annonceur radiophonique ; l'oreille penchée vers l'actualité du journaliste ; un certain instinct didactique qui ne ferait pas honte au professeur; un æil qui, au contact d'innombrables réunions au long des années, perce la dynamique des groupes; enfin le brio du sportif et sa résistance physique aussi bien que ses réflexes rapides; et, avec tout cela, non pas l'esprit du champion, du premier violon ou de la mouche du coche, mais celui de l'assistant, de l'aide qui est là essentiellement pour faciliter le travail des autres et qui s'intègre à l'ensemble d'une équipe.

Mais tout ceci, c'est de la vieille histoire, on le sait depuis toujours... c'est-àdire depuis que le métier d'interprète de conférence a pris suffisamment d'ampleur pour donner matière à réflexion, soit au cours des années 50 . Il est toujours un peu étonnant de redécouvrir combien notre profession est jeune, elle qui semble faire indéracinablement partie du monde moderne! Née des procès de Nuremberg à la fin de la Seconde Guerre mondiale et du foissonnement des organisations internationales peu après, elle a pris du poil de la bête avec la croissance fulgurante des échanges et des communications entraînée par des révolutions électronique et cybernétique. Par le passé, il n'y avait que les rois, les chefs d'armée et les grands diplomates qui avaient dans leur entourage un petit corps d'interprètes. Aujourd'hui, les congrès internationaux réunissent aussi bien les membres du Conseil de sécurité des Nations unies que les cytologues (isolés le reste du temps dans leurs laboratoires-tours d'ivoire) ou les producteurs de volaille, la basse-cour étant devenue agrobusiness. Les interprètes sont de la partie.

Ce qui, évidemment, veut dire qu'ils sont infiniment plus nombreux que jadis. À sa fondation en 1953, l'A.I.I.C. (Association internationale des interprètes de conférence) réunissait la poignée de gens qui venaient d'inventer ce métier en Europe et en jetaient les bases. Cinq ans plus tard, lorsque je me lançais moi aussi sur ces eaux encore pleines de récifs mystérieux, nous étions à peine 250 dans le monde entier. Au Canada, à part une quinzaine d'interprètes permanents à 
l'O.A.C.I. (Organisation de l'aviation civile internationale - membre de la famille des Nations unies), nous étions, Andrée Francour et moi, les deux seules interprètes freelance ou indépendantes - ou, si vous le voulez, "pigistes » selon l'expression étriquée en vogue à Ottawa. Aujourd'hui, que les temps ont changé ! Dans le contexte international d'abord - puisque c'est de là que viennent nos racines - à l'O.A.C.I., la charge de travail a augmenté avec la multiplication des Etats-membres et surtout avec l'adjonction d'une nouvelle langue de travail - le russe - aux trois antérieures : le français, l'anglais et l'espagnol. La multiplication des langues internationales est, d'ailleurs, un des phénomènes de nos jours : de plus en plus nombreuses sont les conférences à 6 et 7 langues, presque inconnues il y a dix ans. Les récents glissements dans l'économie mondiale et dans le poids relatif des détenteurs de ressources a, en particulier, fait de l'arabe une puissante nouvelle venue, et a ajouté une complication de plus à la difficile composition et à l'équilibre précaire des énormes équipes d'interprètes qu'entraîne une telle conférence. En Europe, 'avec ses grands centres d'activité internationale à Genève, Bruxelles, Paris, Londres, Rome et Vienne, la concentration d'interprètes est toujours la plus forte au monde, et ils dépassent aujourd'hui le millier. Dans le reste du monde, des groupes locaux d'interprètes se sont graduellement formés et implantés, d'abord en Amérique du Nord, le groupe canadien ayant tôt opté pour une appartenance démocratiquement mondiale, contrairement à des tendances régionalisatrices et dominatrices chez nos collègues des États-Unis. C'est maintenant en Amérique Latine que la profession d'interprète de conférence s'affine et s'affirme; et petit à petit des groupes en Afrique, en Asie et en Océanie entrent dans le grand courant mondial qui nous unit.

Au Canada, le jeu des nombres est non moins frappant, mais ce n'est pas à cela que se résume l'évolution de la profession d'interprète de conférence. Les chiffres parlent - pas toujours d'eux-mêmes, cependant - il est donc juste de commencer par les regarder. Par rapport au début des années 60 , le nombre d'interprètes permanents a quadruplé, entre quarante et cinquante interprètes et interprètes-traducteurs étant au service du gouvernement fédéral à Ottawa. Là et ailleurs, les indépendants, pendant la même période, ont triplé, si l'on ne compte que ceux qui gagnent leur vie à interpréter : d'une quinzaine, ils sont passés à une cinquantaine. Mais trois autres phénomènes valent la peine qu'on les remarque. Alors qu'il y a dix ans, tous les interprètes indépendants étaient concentrés à Montréal, aujourd'hui de petits groupes de 3 à 5 commencent à apparaître à Toronto, Ottawa et Québec, et prendront assurément plus d'ampleur. Alors qu'il y a dix ans, la quantité de travail était telle qu'elle convenait surtout aux femmes, intéressées à ne pas travailler à temps complet, aujourd'hui un quart ou un cinquième des interprètes indépendants travaillent en une année autant que les permanents, ce qui permet maintenant à des hommes de s'implanter parmi les femmes (quoique entre les permanents, ici comme ailleurs, hommes et femmes sont plus également partagés). Enfin, à part les interprètes dont l'interprétation de conférence est la principale occupation, il y a un nombre croissant, à travers le pays, d'universitaires, d'enseignants ou de professionnels (au sommet de l'échelle) jusqu'à de prétendus «bilingues » (au bas) qui font, à l'occasion, de l'interprétation contre rémunération. Pour répondre à la demande, encore croissante, se multiplient 
également les intermédiaires (écoles de langues, entreprises d'installation de matériel audiophonique) qui offrent leurs services à rabais et dans des conditions souvent trompeuses pour le client inaverti.

C'est justement au scin de la «clientèle », au Canada, qu'il y a eu le moins de changement. Les nombres ont, évidemment, grandi. Mais en général, les clients sont toujours aussi indulgents, satisfaits, émerveillés de voir des sons étrangers sortir des cabines à vitres doubles par l'enchevêtrement de fils, microphones et autres gadgets. À moins qu'ils ne soient franchement irrités par ces entraves inutiles au bon fonctionnement des réunions où tous s'entendent parfaitement en anglais de toute manière...

Mais, pardon. Si changement il y a, c'est surtout parmi les fonctionnaires fédéraux anglophones, où un certain nombre, lors de conférences qui leur donnent le loisir de somnoler, préfèrent se distraire en écoutant l'interprétation française pour prolonger leurs cours «d'immersion». Et la politique linguistique d'Ottawa leur en donne, c'est sûr, de plus en plus l'occasion. Ainsi, parmi les hauts fonctionnaires, il y a un nombre grandissant qui peuvent dire, en un français honorable, les quelques mots de mise à l'ouverture d'une séance (point besoin d'interprétation, nul n'écoute si ce n'est que tous comprennent). Quant aux Québécois francophones, s'il est vrai qu'une plus forte proportion d'hommes politiques, de technocrates ou de cadres n'est plus, aujourd'hui, aussi parfaitement bilingue qu'elle l'était naguère, ils ne savent pas plus aujourd'hui qu'hier comment s'y prendre pour pouvoir se fier à l'interprétation s'ils désirent s'exprimer en français. Alors, plutôt que de courir le risque d'être mal compris, ils préfèrent s'imposer l'anglais, qu'ils manient parfois brillamment, parfois boîteusement. Ils n'ont pas d'autre choix - dommage, n'est-ce pas ?

La multiplication trop rapide des interprètes, liée évidemment à la demande n'est pas étrangère au problème. Dans toute profession, il y a les bons et les moins bons, caveat emptor. Mais dans celle-ci, les pressions du marché ont été si fortes au cours des dernières années, qu'il y a une proportion démesurée de « débutants », réels ou éternels, qui réussissent à survivre et même à faire bonne fortune - généralement aux frais des contribuables, car les gens qui dépensent l'argent de leur poche s'arrangent pour mieux savoir ce qu'ils en tirent.

Une fois perçus les maux, il est plus aisé de concevoir les remèdes. Les états chroniques ne guérissent pas en un seul jour ; mais c'est aux intéressés de prendre les moyens voulus pour mettre la convalescence en marche.

L'intéressé au premier rang, c'est celui qui organise ou qui assiste à une réunion bilingue dont il sait qu'elle aura besoin d'interprétation. Eh bien, il n'y a pas de recette magique en trois lignes qui lui révèlera tous les secrets de coulisse... Il faudra qu'il s'instruise pour bien s'y prendre d'avance ; qu'il aille chercher les avis de personnes compétentes... Ce n'est pas tout le monde qui traite d'affaires aussi importantes que Charles de Gaulle lorsqu'il rencontrait d'autres chefs d'État - mais il avait son interprète et refusait systématiquement de se fier à ceux des autres... À moindre échelle, l'exemple vaut peut-être la peine d'être retenu. Il s'agit d'apprendre à connaître les interprètes à qui l'on veut faire confiance. 
Il a été question plus haut des pressions du marché. La demande a effectivement été très forte, ces dernières années surtout à la suite de la loi fédérale sur les langues officielles qui exige le bilinguisme des organes fédéraux et encourage celui de toute association canadienne. La loi a eu des répercussions importantes sur la quantité tant des traductions que de l'interprétation fournie ou commanditée par le gouvernement. Il ne m'appartient pas de juger de l'effet que cette augmentation soudaine a eu sur la qualité des travaux de traduction, mais les écrits étant relativement permanents, c'est une donnée objectivement véritable. Le côté éphémère de l'interprétation rend son appréciation beaucoup plus difficile. Ce n'est qu'à l'expérience répétée que l'on se rend compte combien une demande «artificielle », dans le sens qu'elle ne répond qu'à des exigences purement formelles, peut détériorer la qualité de la prestation. La réunion doit être bilingue (c'est la loi), par conséquent il faut faire les démarches nécessaires pour obtenir l'interprétation, c'est la clé de tout, se disent les responsables. On trouve les fonds, la cabine, des interprètes - voilà le tour joué ! Tout le monde est content. Personne ne se soucie de savoir si quelqu'un dans la salle en a vraiment besoin. Si oui, tant pis pour lui, il y a de bonnes chances que l'on n'ait prévu ni la préparation nécessaire des interprètes, ni une composition soignée de l'équipe, ni des conditions sur place qui favoriseront une bonne interprétation. Marchera, marchera pas ? C'est à voir, et ce sera à l'auditeur d'en juger. Si ça marche, ce n'est que le cours normal des choses. Si ça ne marche pas, l'auditeur sera bien trop poli, ou trop peu enclin à faire le troublefête, pour s'en plaindre aux organisateurs, ou même pour en glisser mot aux interprètes.

Pire encore, dans de trop nombreux cas, l'interprétation n'est là que pour la forme, personne n'en ayant besoin. La loi est la loi et c'est la Reine qui paye, pourquoi s'en faire? Effectivement, personne ne s'en fait, et bien du monde en profite. Les interprètes ne travaillent pas plus fort ni mieux que d'habitude: lorsque personne n'écoute, plus de distinction entre l'ancien et le débutant, entre le francophone, l'anglophone ou l'alingue en cabine, entre le fort en thème et le cancre... Cela perpétue le laisser-aller, l'indifférence, lâchons le grand mot, l'incompétence. Bien difficile de sortir du cercle vicieux lorsque subitement l'usager, le vrai, réapparaît dans le cadre...

En fin de compte, c'est aux organisateurs de réunions de prendre leurs responsabilités au sérieux, d'exiger un bon service d'interprétation lorsqu'il est nécessaire pour des participants en chair et en os. Et c'est à ceux-ci de manifester clairement leurs besoins. Ni les réunions, ni le bilinguisme ne sont bien servis par la fiction qui se crée lorsque l'interprétation ne répond qu'à des besoins hypothétiques et incorporels, lorsque l'on se contente de satisfaire à la lettre et non pas à l'esprit de la loi. Ce n'est pas en y mettant plus de dollars que l'on corrigera ces anomalies-là.

Mais, direz-vous enfin, qu'en est-il des interprètes en cause ? N'ont-ils pas un rôle à jouer eux aussi dans l'assainissement de la situation ? C'est un peu le même phénomène que de se plaindre des retardataires aux gens qui arrivent à temps. Ceux parmi nos collègues-interprètes qui accepteront le dialogue à ce sujet ont déjà acquis une conscience professionnelle et le respect du code d'éthique et des normes de travail acceptées tant au sein de l'A.I.I.C. que bien au-delà de ses cadres. Les associations, corporations ou ordres professionnels ont, évidemment, 
des moyens de pression et d'action auprès de leurs membres - mais se dit interprète qui veut; peu de gens savent que cela ne le rend pas automatiquement apte à accéder aux rangs de l'A.I.I.C.

D'autre part, il est bien vrai qu'une profession consciente de ses responsabilités s'occupe également de la qualité de la formation offerte à ses futurs membres; elle est soucieuse d'ouvrir grandes les portes d'accès aux éléments les plus doués. Ainsi, les membres de l'A.I.I.C. jouent un rôle actif dans les cours offerts à l'Université de Montréal dans le cadre de la licence, et de la maîtrise en traduction avec option interprétation. Et ils réfléchissent à la possibilité, inédite encore, de mettre au point et d'offrir, dans un avenir rapproché, des ateliers de perfectionnement ou de recyclage à des interprètes en exercice. Nous avons déjà, au Québec, des interprètes de réputation mondiale, d'un calibre égal aux meilleurs que l'on trouve n'importe où ailleurs. Ces mesures aideront, osons l'espérer, à combler l'écart qui subsiste encore entre ce petit nombre et les besoins réels du milieu.

THÉRÈSE ROMER 